\title{
Three-dimensional flow simulations for polymer extrudate swell out of slit dies from low to high aspect ratios
}

Cite as: Phys. Fluids 31, 093103 (2019); https://doi.org/10.1063/1.5116850

Submitted: 30 June 2019 . Accepted: 03 September 2019 . Published Online: 19 September 2019

Dahang Tang (D), Flavio H. Marchesini (D), Ludwig Cardon, and Dagmar R. D'hooge (D)

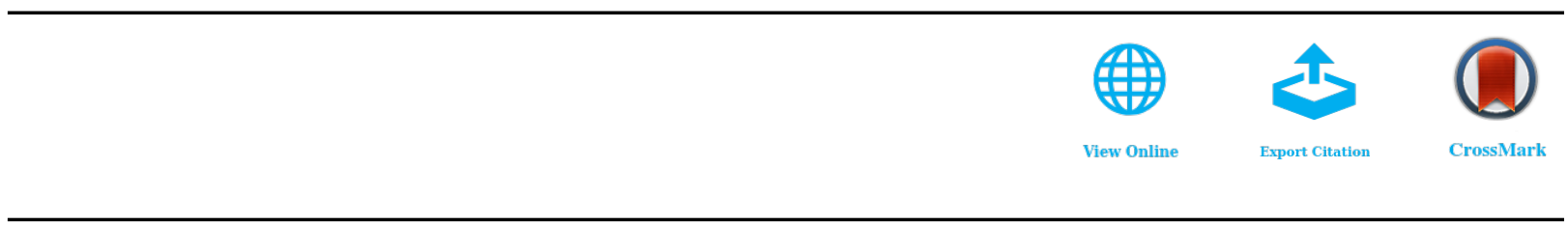

\section{Scilight Highlights of the best new research} in the physical sciences 


\title{
Three-dimensional flow simulations for polymer extrudate swell out of slit dies from low to high aspect ratios
}

\author{
Cite as: Phys. Fluids 31, 093103 (2019); doi: 10.1063/1.5116850 \\ Submitted: 30 June 2019 - Accepted: 3 September 2019 • \\ Published Online: 19 September 2019
}

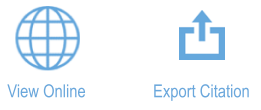

\author{
Dahang Tang,' (D) Flavio H. Marchesini,' (D) Ludwig Cardon,' and Dagmar R. D'hooge, \\ AFFILIATIONS \\ ${ }^{1}$ Centre for Polymer and Material Technologies, Department of Materials, Textiles and Chemical Engineering, Ghent University, \\ Technologiepark 130, 9052 Zwijnaarde, Ghent, Belgium \\ ${ }^{2}$ Laboratory for Chemical Technology, Department of Materials, Textiles and Chemical Engineering, Ghent University, \\ Technologiepark 125, 9052 Zwijnaarde, Ghent, Belgium \\ ${ }^{3}$ Centre for Textile Science and Engineering, Department of Materials, Textiles and Chemical Engineering, Ghent University, \\ Technologiepark 70a, 9052 Zwijnaarde, Ghent, Belgium
}

a) Author to whom correspondence should be addressed: dagmar.dhooge@ugent.be

\begin{abstract}
The impact of the slit die geometry and the polymer melt flow characteristics on the extrudate swell behavior, which is a key extrusion operating parameter, is highlighted. Three-dimensional (3D) numerical simulations based on the finite element method are compared with their conventional two-dimensional (2D) counterparts at the same apparent shear rates using ANSYS Polyflow software. The rheological behavior is described by the differential multimode Phan-Thien-Tanner constitutive model, with polypropylene as a reference. It is shown that increasing the aspect ratio of the die geometry (width/height ratio variation from 1 to 20 ) contributes to a significant change in the $3 \mathrm{D}$ extrudate deformation (relative changes of $10 \%$ in several directions; absolute changes up to $30 \%$ ) and delays the equilibrium axial position (up to a factor 10). High aspect ratios induce a switch to contract flow (swell ratio $<1$ ) for the edge height swell. The $3 \mathrm{D}$ extrudate swell strongly deviates from the 2D simplified case due to the die effect near the wall, even for higher aspect ratios. Also a different relation with the material parameters is recorded. The initially large swell behavior is followed by a small shrinkage flow in the middle height direction which cannot be captured by the $2 \mathrm{D}$ counterpart. The findings are supported by a comprehensive analysis of the velocity and stress fields in and out of the slit dies.
\end{abstract}

Published under license by AIP Publishing. https://doi.org/10.1063/1.5116850

\section{INTRODUCTION}

Extrusion is a polymer forming operation under melt conditions that is widely applied for industrial applications. ${ }^{1-5}$ It is usually observed that the dimensions of the extrudate coming out of the extruder are larger than the dimensions of the extruder orifice. This phenomenon is denominated as extrudate or die swell and is a key process control parameter in view of product stability. ${ }^{6}$ Investigating the swell behavior upon flow exit only through experimental methods is not optimal and time consuming. ${ }^{7,8}$ Hence, to obtain additional insights, numerical flow simulations are recommended. Such simulations have the advantage of tracking the varying trend of velocity and stress fields for different processing conditions. ${ }^{\text {- }}$
The choice of the constitutive equation to grasp the viscoelastic properties of the fluids is essential. Early studies were carried out using the basic Maxwell viscoelastic constitutive model. ${ }^{13}$ However, this model cannot capture realistic or nonlinear viscoelastic properties of polymer fluids, such as shear-thinning and elongationthinning behavior in the high strain rate region. ${ }^{14}$ Some more realistic constitutive models have therefore been proposed, such as the integral Kaye-Bernstein-Kearsley-Zapas (KBKZ) constitutive model, ${ }^{15-19}$ the differential Phan-Thien-Tanner (PTT) model, ${ }^{20-}$ and the Giesekus model. ${ }^{25,27}$ Among them, the integral KBKZ model and the differential PTT model seem suited due to the possibility of describing the shear and elongational properties of polymer melts independently. ${ }^{14,28-30}$ 
A variety of geometrical characteristics, including the die entrance angle, the length/diameter (or thickness) ratio, the width/thickness ratio, and the outer/inner diameter ratio, have already theoretically been shown to play an important role in determining the final thus equilibrated extrudate swell behavior. $^{23,24,27,31-33}$ For example, García-Rejón et al. ${ }^{32}$ systematically studied the effect of the channel geometry and inclination angle on the annular extrudate swell from the diverging, converging, and straight annular die, considering the integral constitutive $\mathrm{KBKZ}$ model for two-dimensional (2D) isothermal high density polyethylene (HDPE) flow. They concluded that both the thickness and diameter swell are very sensitive to the die geometry. Mu et al. ${ }^{24}$ simulated in detail the impact of the die geometrical parameters on the 3D annular extrudate swell for isothermal low density polyethylene (LDPE) flow, including the effect of the die contraction angle, the ratio of parallel length to inner radius, and the ratio of outer to inner radius. More recently, Hatzikiriakos et al. ${ }^{31,33,34}$ conducted 2D simulations with varying length/diameter and width/height (or equivalently width/thickness) ratio mainly with the KBKZ model to figure out the relevance of the die geometry. These authors indicated that increasing the length/diameter ratio decreases the extrudate swell in agreement with their experimental results.

It should be realized that in many cases, the $3 \mathrm{D}$ flow pattern during and after extrusion is simplified into isothermal 2D flow to lower the computational cost and in favor of numerical convergence. $^{26,33,35-38}$ For example, for planar flow configurations, 2D simulations imply an infinite width/height $(W / H)$ or aspect ratio. However, the aspect ratios of flow channels are in reality finite and thereby, a deviation is expected between the experimental observations and 2D simulations, which is referred to as the $3 \mathrm{D}$ or edge effect. ${ }^{28,39-42}$ For example, Clemeur et al. ${ }^{40}$ showed a mismatch with the observed isochromatic birefringence of a polymer solution in slit dies for the simplified isothermal 2D simulations with varying aspect ratio, which enlarged by increasing the flow rate. Schoonen ${ }^{28,43}$ found that decreasing the aspect ratio for polyisobutylene (PIB) flow in a rectangular flow channel from 8 to 2 resulted in an increase in the discrepancy between isothermal $2 \mathrm{D}$ and 3D simulations. For instance, the difference between the simulated first normal stress difference of the PIB solution and that of the $2 \mathrm{D}$ one at the same apparent shear rate is only $11 \%$ for an aspect ratio of 8 but increases to $30 \%$ for an aspect ratio of 2 .

Nevertheless, the 3D effect on the polymer extrudate swell behavior is typically not studied, as one focuses mainly on the flow at the end of the extruder equipment. In our recent work, ${ }^{44}$ we carried out comprehensive isothermal 3D simulations of the extrudate swell behavior of neat polypropylene (PP) flow from a slit die at $200^{\circ} \mathrm{C}$ with fixed dimensions (aspect ratio of 10). We highlighted that the distance from the die exit, within which the final extrudate shape is obtained, strongly depends on the swell behavior in the width direction, indicating that $3 \mathrm{D}$ effects must be considered for optimization of the die design. Moreover, the die swell simulations results were experimentally confirmed by $3 \mathrm{D}$ imaging, considering experimentally verified PTT input model parameters.

In the present study, we further focus on the dependence of PP extrudate swell behavior on the geometric characteristics, specifically the aspect ratio of the slit die. Consequently, 3D simulations of PP melt with different aspect ratios have been conducted using the PTT model as previously tuned. The computational results are compared to those obtained from the corresponding 2D simulations with the aim at assessing the relevance of the $3 \mathrm{D}$ effect. A significant discrepancy of the swell behavior between the two modeling configurations is reported. The corresponding flow velocity and stress fields are further discussed in detail to illustrate the mechanism for this discrepancy. A parametric study regarding the key parameters of the PTT model is additionally included to clarify the influence of the polymer rheological properties on the $3 \mathrm{D}$ effect of the flow channels.

\section{MODELING PRINCIPLES}

\section{A. Geometric parameters}

Figure 1 depicts the 2D schematic diagram of the cross section of a slit die with an aspect ratio of 10 . Several swell ratios can be defined as the ratios between the dimensions of the extrudate and the die. To identify a possible anisotropic extrudate swell behavior, three such ratios $B_{1}, B_{2}$, and $B_{3}$ are needed, denoting the swelling in the extrudate width, edge height, and middle height direction (Fig. 1), respectively,

$$
B_{1}=\frac{L_{- \text {extrudate }}}{L_{- \text {die }}}, B_{2}=\frac{D_{1-\text { extrudate }}}{D_{1-\text { die }}} \text {, and } B_{3}=\frac{D_{2-\text { extrudate }}}{D_{2-\text { die }}} .
$$

Additionally, $B_{4}$ is defined as the swell ratio of the area size (S) of the extrudate cross section and the slit die,

$$
B_{4}=\frac{S_{- \text {extrudate }}}{S_{- \text {die }}} \text {. }
$$

\section{B. Governing equations}

In this work, we assume that the incompressible polymer fluid is under steady and creeping laminar flow conditions $\left(200^{\circ} \mathrm{C}\right)$ without inertial force, gravity, and surface tension effects. The applied governing equations include the following continuity and momentum equations in vector notation: ${ }^{44,45}$

$$
\begin{gathered}
\nabla \cdot \mathbf{v}=0, \\
-\nabla p+\nabla \cdot \boldsymbol{\tau}=0,
\end{gathered}
$$

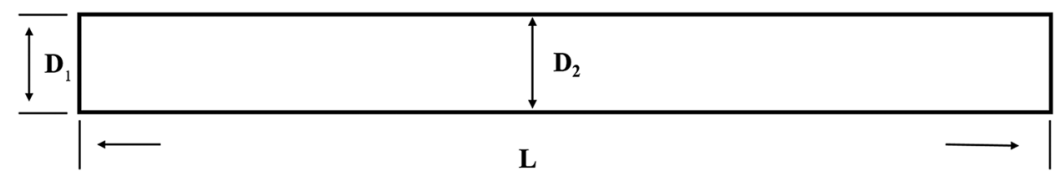

FIG. 1. The cross section of a slit die (case of aspect ratio of 10). Four swell ratios are defined, as mathematically represented by Eqs. (1) and (2). They relate to swelling in the width direction, the edge height direction, and the middle height direction. Area swelling is also included. 
TABLE I. PTT model for PP melt flow at $200^{\circ} \mathrm{C} .^{44}$ In Fig. 2, the relevance of this model is highlighted by a sensitivity analysis of the nonlinear material parameters $\xi_{3}$ and $\varepsilon_{3}$.

\section{PTT model}

\begin{tabular}{lccccccccl}
\hline Material & Mode & $\lambda_{\mathrm{i}}(\mathrm{s})$ & \multicolumn{3}{c}{$\eta_{\mathrm{i}}(\mathrm{Pa} \mathrm{s})$} & & $\varepsilon_{\mathrm{i}}$ & & $\xi_{\mathrm{i}}$ \\
\hline Neat PP & 1 & $\lambda_{1}$ & 0.01 & $\eta_{1}$ & 536 & $\varepsilon_{1}$ & 0.17 & $\xi_{1}$ & 0.16 \\
& 2 & $\lambda_{2}$ & 0.1 & $\eta_{2}$ & 816 & $\varepsilon_{2}$ & 0.18 & $\xi_{2}$ & 0.2 \\
& 3 & $\lambda_{3}$ & 1 & $\eta_{3}$ & 707 & $\varepsilon_{3}$ & 0.27 & $\xi_{3}$ & 0.2 \\
& 4 & $\lambda_{4}$ & 10 & $\eta_{4}$ & 220 & $\varepsilon_{4}$ & 0.4 & $\xi_{4}$ & 0.3 \\
\hline
\end{tabular}

in which $v$ is the velocity vector, $p$ is the hydrostatic pressure, and $\tau$ is the extra stress tensor. For the polymer melts, the constitutive model associating the extra stress with the velocity or velocity gradients is essential to solve the set of equations.

The extra stress tensor for the viscoelastic constitutive model, $\boldsymbol{\tau}$, is split into a purely viscous part, $\tau_{N}$, and a viscoelastic part, $\tau_{p}$. $\tau_{N}$ is solved by the generalized Newtonian flow model, ${ }^{44}$ while $\tau_{p}$ is calculated by the basic viscoelastic constitutive equations in terms of the differential (or integrated) constitutive model. Regarding a time spectrum of $N$ relaxation modes, the viscoelastic stress tensor, $\boldsymbol{\tau}_{p}$, is calculated as a sum of $N$ individual $\tau_{p i}$ contributions,

$$
\tau_{p}=\sum_{i=1}^{N} \tau_{p i}
$$

Each individual contribution $\tau_{p i}$ is revealed by the PTT constitutive equation as it shows a good ability to describe the rheological properties of PP melt including the shear-thinning and extensionalthinning behavior in the high strain rate range as demonstrated in our previous work. ${ }^{44}$ The PTT model is written as

$$
\exp \left[\frac{\varepsilon_{i} \lambda_{i}}{\eta_{i}} \operatorname{tr}\left(\tau_{p i}\right)\right] \tau_{p i}+\lambda_{i}\left[\left(1-\frac{\xi_{i}}{2}\right) \overline{\tau_{p i}}+\frac{\xi_{i}}{2} \widehat{\tau_{p i}}\right]=2 \eta_{i} D,
$$

in which $\lambda_{\mathrm{i}}$ and $\eta_{\mathrm{i}}$ are the relaxation time and shear viscosity, determining the linear rheological properties. The nonlinear parameters, $\varepsilon_{i}$ and $\xi_{i}$, control the extensional and shear behavior. $\overline{\tau_{p i}}$ and $\widehat{\boldsymbol{\tau}_{p i}}$ correspond to the upper and lower convected time derivatives of $\boldsymbol{\tau}_{p i}$. $\boldsymbol{D}$ represents the deformation tensor rate, which is defined as

$$
\boldsymbol{D}=\frac{\nabla \boldsymbol{v}+(\nabla \boldsymbol{v})^{T}}{2}
$$

with $\nabla \boldsymbol{v}$ and $(\nabla \boldsymbol{v})^{T}$ being the velocity gradient and transpose of the velocity gradient.

For the PP in this study, the material parameters of the multimode PTT constitutive model are extracted from the good fitting of the linear and nonlinear rheological properties, as described in our previous work. ${ }^{44}$ The corresponding parameters are shown in Table I, which are comparable to a previous report. ${ }^{46}$ For illustration purposes, a sensitivity analysis of the nonlinear material parameters $\xi_{3}$ and $\varepsilon_{3}$ of the PTT model regarding rheological properties is shown in Fig. 2. In comparison with the parameter $\xi_{3}$, increasing $\varepsilon_{3}$ not only increases the first normal stress difference $N_{x y}\left(\tau_{x x}-\tau_{y y}\right)$ but also significantly increases the elongational viscosity. On the other way, $\xi_{3}$ mainly has a significant influence on the second normal stress difference $N_{y z}\left(\tau_{y y}-\tau_{z z}\right)$ component.

\section{General geometry and boundary conditions}

The $3 \mathrm{D}$ flow domain for a given $W / H$ ratio is shown in Fig. 3. Due to the symmetry, only one quarter of the flow channel is considered for all simulations to reduce the computational cost. The
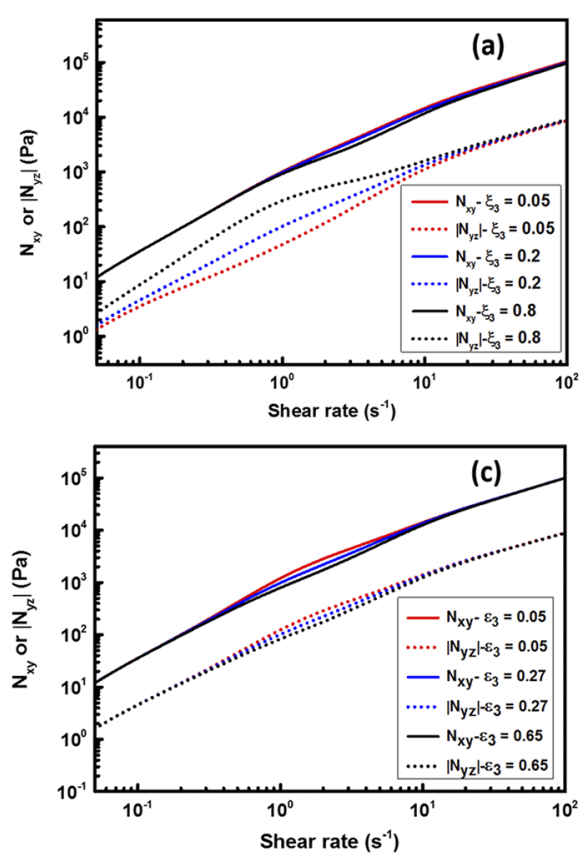
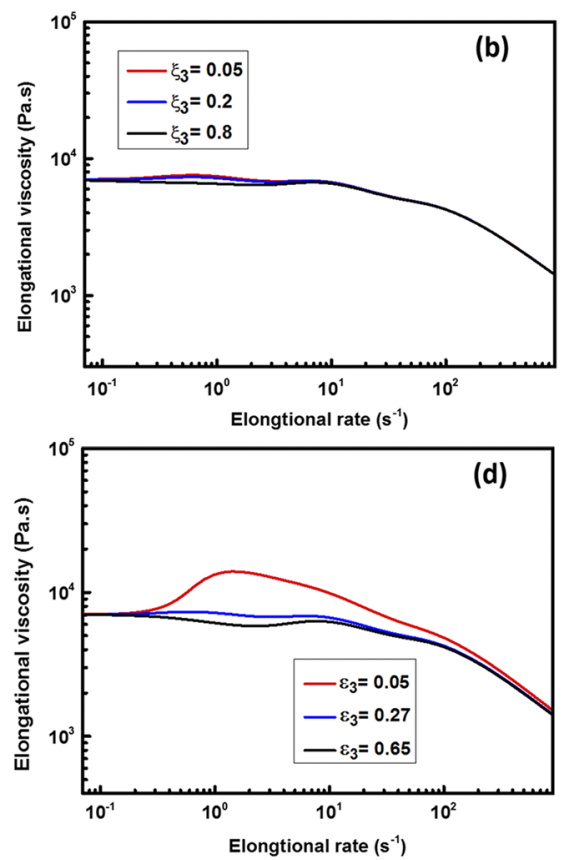

FIG. 2. Effect of the material parameter $\xi_{3}\left[(a)\right.$ and (b)] and $\varepsilon_{3}[(\mathrm{c})$ and (d)] on the normal stress difference $N_{x y}$ and $\left|N_{y z}\right|$ and the elongational viscosity as functions of shear and elongational rate; reference parameters are given in 


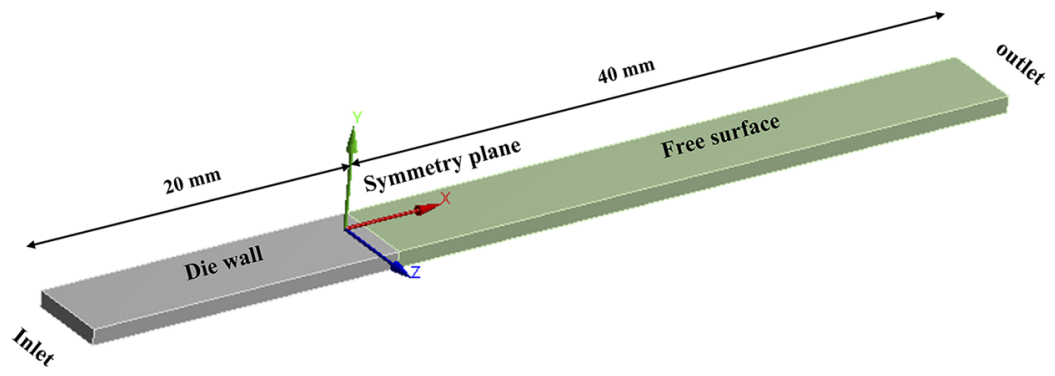

FIG. 3. 3D flow domain of a slit die profile (see Table II). One quarter is considered for all simulations due to symmetry $\left(I_{1}=20 \mathrm{~mm}\right.$ and $\left.I_{2}=40 \mathrm{~mm}\right)$.

geometrical parameters for different flow channels (varying aspect ratio) are shown in Table II. The general boundary conditions are set as reported in previous work. ${ }^{44}$ At the inlet, a fully developed flow is assumed due to the long die length for the experimental comparison $(160 \mathrm{~mm}$; aspect ratio of 10). At the die walls, a nonslip condition for the velocity field is prescribed, which indicates that the flow velocity vanishes: $v_{n}=v_{t}=0(\mathrm{~mm} / \mathrm{s})$. Outside the die exit, a free surface flow is developed, which is unknown a priori. The force conditions $f_{t}=f_{n}=0(N)$ are set at the outlet of the flow domain, with the subscript $n$ and $t$ representing the normal and tangential component of the force and velocity.

\section{Method of 2D and 3D numerical solution for various aspect ratios}

To solve the conservation and constitutive equations above, a flow solver (POLYFLOW version, ANSYS version 18) is applied based on the finite element algorithm. A discrete elastic viscous stress splitting (DEVSS) combined with the streamline-upwind (SU) scheme is used to improve the numerical calculation stability. $933,37,42,47$ This is done as there is a geometric singularity near the die exit where an abrupt change for the flow occurs from the nonslip to the free surface boundary condition. The DEVSS method adds a purely viscous term into the momentum equation based on velocity unknowns and removes an equivalent contribution based on rate-ofdeformation unknowns to maintain the constitutive equation. ${ }^{48}$ In addition, we apply an interpolation method on the velocity and pressure by quadratic and linear functions. An evolutionary scheme is performed on the volumetric flow rate to advance the solution from almost purely Newtonian to the viscoelastic flow with high nonlinear rheological properties.
The height of each slit die is fixed at $2 \mathrm{~mm}$, and the aspect ratio includes values of $1,1.5,2,5,10$, and 20 (see Table II). The mesh sizes used in both $x$ and $y$ coordinates (shown in Fig. 3) of the geometry model with different aspect ratios are maintained at the same level in all 3D simulations. ${ }^{40,42,49}$ A mesh grid that progressively adds more elements toward the exit singularity is utilized, while larger mesh elements are applied in the flow region away from the singularity. ${ }^{50}$ These elements are shown in Fig. S1 in the supplementary material. The analysis of the mesh independence for the swell simulations can be found in Table SI in the supplementary material.

To quantify the effect of the die geometry on the flow behavior, the results obtained from different geometry configurations are compared at a constant $\dot{\gamma}_{\mathrm{a}}$ value as in the work of Clemeur et al. ${ }^{37}$ Generally, the dimensionless Weissenberg number $W i$ is adopted to evaluate the viscoelasticity level involved in polymer flows,

$$
W i=\dot{\gamma}_{a} \lambda_{m}
$$

in which $\lambda_{m}$ is the viscoelastic average relaxation time for the polymer fluid,

$$
\lambda_{m}=\frac{\sum_{i=1}^{N} \eta_{i} \lambda_{i}}{\sum_{i=1}^{N} \eta_{i}},
$$

and $\dot{\gamma}_{a}$ is an apparent shear rate in the slit die with no slip,

$$
\dot{\gamma}_{a}=\frac{6 Q}{2 W *(2 H)^{2}} \text {. }
$$

It should be noted that Eq. (10) works well only if $W / H$ is larger than $10,^{37,52}$ which is thus not the case for most considered aspect ratios in the present work. In contrast, if the aspect ratio decreases to smaller values such as 1 and 2, this equation is too approximated to calculate

TABLE II. Geometrical parameters for flow channels with various aspect ratios; link to Fig. 2; $Q_{1}$ and $Q_{2}$ correspond to $\dot{\gamma}_{\mathrm{a}}=15$ and $48 \mathrm{~s}^{-1}$, which are maintained as target values in line with the work of Clemeur et al. ${ }^{37}$

\begin{tabular}{lcccccc}
\hline \hline $\begin{array}{l}\text { Aspect } \\
\text { ratio }(W / H)\end{array}$ & $\begin{array}{c}\text { Width } \\
(2 W)(\mathrm{mm})\end{array}$ & $\begin{array}{c}\text { Height } \\
(2 H)(\mathrm{mm})\end{array}$ & $\begin{array}{c}l_{1} \\
(\mathrm{~mm})\end{array}$ & $\begin{array}{c}l_{2} \\
(\mathrm{~mm})\end{array}$ & $\begin{array}{c}Q_{1} \\
\left(\mathrm{~mm}^{3} / \mathrm{s}\right)\end{array}$ & $\begin{array}{c}Q_{2} \\
\left(\mathrm{~mm}^{3} / \mathrm{s}\right)\end{array}$ \\
\hline 1 & 2 & 2 & 20 & 40 & 13 & 42 \\
1.5 & 3 & 2 & 20 & 40 & 22 & 69 \\
2 & 4 & 2 & 20 & 40 & 31 & 98 \\
5 & 10 & 2 & 20 & 40 & 91 & 288 \\
10 & 20 & 2 & 20 & 40 & 196 & 620 \\
20 & 40 & 2 & 20 & 40 & 405 & 1280 \\
\hline \hline
\end{tabular}


$\dot{\gamma}_{a}$. Therefore, for each simulation case where formally Newtonian flow is extruded from a slit die with a certain $W / H$ ratio, the flow rate value applied in the Polyflow software is modified to make sure that the final apparent shear rates are the same. The values of 15 and $48 \mathrm{~s}^{-1}$ are considered for $\dot{\gamma}_{a}$ (see last two columns in Table II for $Q$ values).

For the simplified 2D pattern, the discretization of the mesh pattern in the $x-y$ plane is the same as that of all 3D counterparts to avoid some difference only arising from the mesh discretization (Fig. S2 in the supplementary material). ${ }^{37,40}$ The interpolation methods for all unknown physical fields such as velocity and pressure variables for all $2 \mathrm{D}$ and $3 \mathrm{D}$ cases are the same.

To further demonstrate the relevance of the simulation results, focus is also on the comparison with the analytical solution proposed by Tanner to evaluate the swell ratio of the $2 \mathrm{D}$ simplified planar viscoelastic flow based on stress analysis, ${ }^{53,54}$

$$
B_{3}=0.19+\left(1+\frac{S_{w}{ }^{2}}{3}\right)^{1 / 4}
$$

in which $S_{w}$ indicates a half of the ratio of the first normal stress difference to the shear stress on the die wall. The swell ratio predicted with Eq. (11) plugging in the simulated value of $S_{w}$ is around 1.28 at $\dot{\gamma}_{\mathrm{a}}=48 \mathrm{~s}^{-1}$. This is slightly smaller than the numerically simulated value of 1.31 for the simplified $2 \mathrm{D}$ flow in this study and can be related to the ignoring of the stress singularity in Tanner's equation. This equation is only based on a physical analysis of the die-swell problem, with a combination of two characteristic properties of viscoelastic materials: recoiling and the existence of the normal stress difference. ${ }^{53}$ Overall, the good match indicates the reasonability of the numerical simulations in this study for sure in the context of identifying trends regarding the relevance of the aspect ratio.

\section{RESULTS AND DISCUSSION}

\section{A. Effect of aspect ratio on the 3D die swell characteristics}

An important characteristic of the PP extrudate swell behavior is the continuous evolution when the flow emerges the die exit. ${ }^{18}$ Figure 4 depicts the variations of the predicted extrudate profiles with an aspect ratio range from 1 to 20 at $\dot{\gamma}_{\mathrm{a}}=48$ (top row) and 15 (bottom row) $\mathrm{s}^{-1}$. The associated $Q$ values are given in Table II. For the aspect ratio of 10 , previously recorded experimental data have been included as well. ${ }^{44}$ Good agreement between simulation and experimental results is observed. As shown in Figs. 4(a) and 4(c), increasing the aspect ratio results in a decrease in the width swell ratio $B_{1}$ but a larger distance to obtain an equilibrium swelling. In Figs. 4(b) and 4(d), the evolution of the swelling behavior of the extrudate edge height (swell ratio $B_{2}$ in Fig. 1) is compared, as only accessible with $3 \mathrm{D}$ simulations. It is shown that a higher aspect ratio is able to strongly change the flow pattern along the extrudate edge height ultimately with "negative" swelling or contract flow $\left(B_{2}<1\right)$. For an aspect ratio of 1 , a significant swelling is noted since the $B_{2}$ value sharply increases to around 1.15 , which seems similar to the results for linear low density polyethylene (LLDPE) extrudate from a cubic die. ${ }^{55}$ Increasing slightly the aspect ratio from 1 to 1.5 leads to a significant drop in the $B_{2}$ value. As the aspect ratio increases further $(5,10$, and 20$)$, a contract flow in the edge height direction is observed.

In Fig. 5, the corresponding changes in the middle height swell ratio $B_{3}$ are plotted (symbols) against the axial distance $\left[\dot{\gamma}_{\mathrm{a}}=48\right.$ (top row) and 15 (bottom row) $\mathrm{s}^{-1}$ ]. Clearly, the values are different from the $B_{2}$ values. Anisotropy of extrudate swell behavior is thus observed in all directions (see Table III). A comparison with the die swell behavior of the corresponding "ideal" 2D flow is depicted as
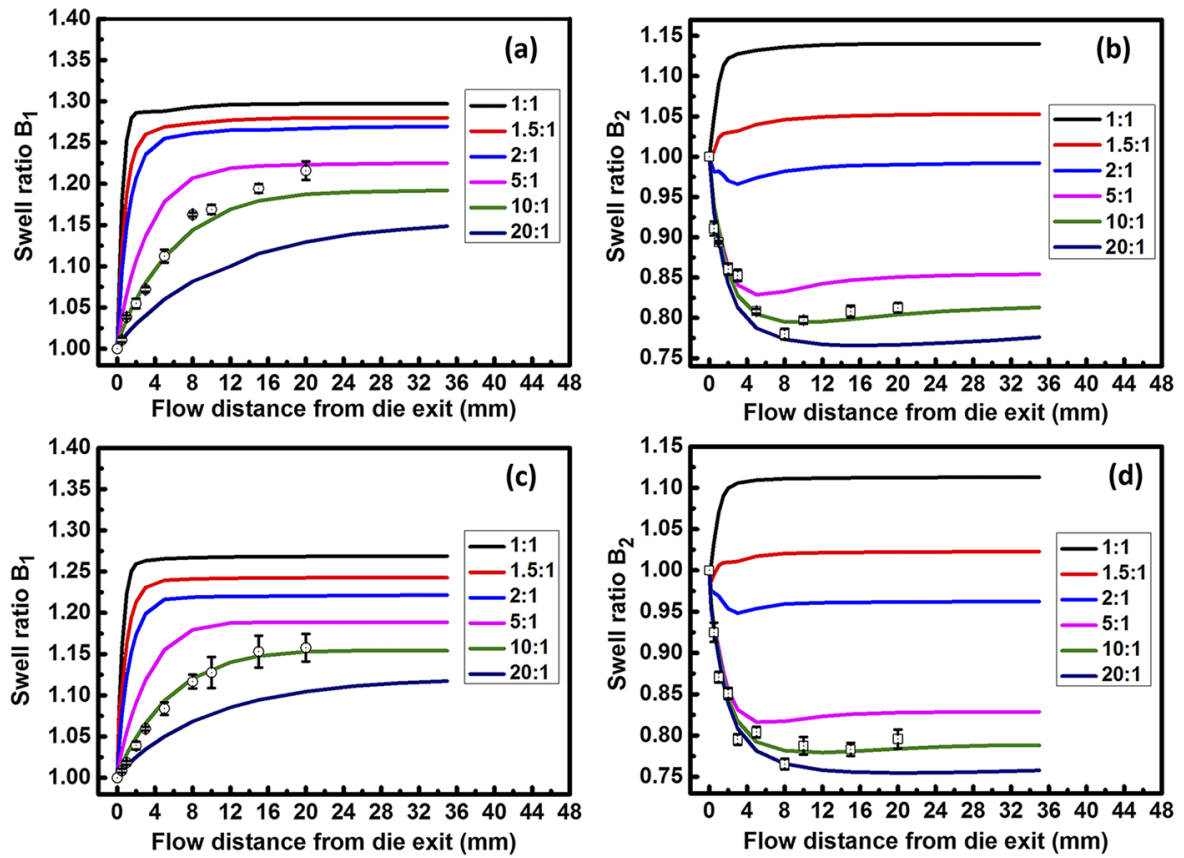

FIG. 4. Comparison of the predicted extrudate profiles through slit dies with different aspect ratios (from 1:1 to 20:1; W/H; $200^{\circ} \mathrm{C}$; Table II) at $\dot{\gamma}_{\mathrm{a}}=48 \mathrm{~s}^{-1}$ (top) and $15 \mathrm{~s}^{-1}$ (bottom): width swell ratio $B_{1}[(a)$ and $(c)]$ and edge height swell ratio $B_{2}[(\mathrm{~b})$ and (d)] [Eq. (1); PTT parameters from Table I]. Symbols indicate the experimental data for an aspect ratio of 10. 

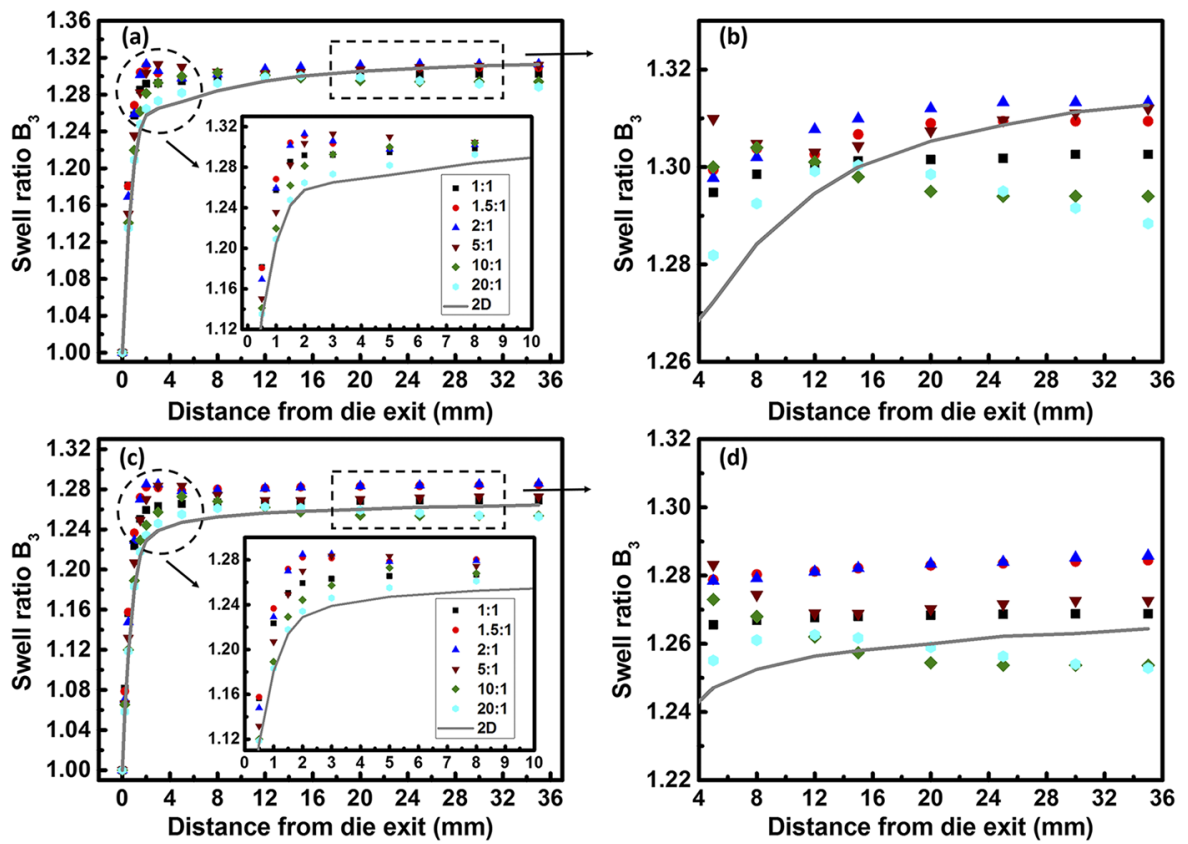

FIG. 5. (a) Evolution of the predicted middle height swell ratio $B_{3}[$ [Eq. (1)] of PP extrudate $\left(200^{\circ} \mathrm{C}\right.$; Table II) for the simplified 2D (line) and 3D flows (symbols) at $\dot{\gamma}_{\mathrm{a}}=48 \mathrm{~s}^{-1}$ [top row; (a) full image; (b) zoom in] and $\dot{\gamma}_{\mathrm{a}}=15 \mathrm{~s}^{-1}$ [bottom row; (c) full image; (d) zoom in]; PTT parameters are from Table $\mathrm{l}$. well in Fig. 5 (gray lines). A larger aspect ratio results in a smaller final equilibrium height swell ratio $B_{3}$, which is similar to the results of Konaganti et al. ${ }^{33}$ In a short distance downstream the die exit, significant deviations between the 2D and 3D simulations are obtained, as made even clearer in the lower graphs. Specifically, the $3 \mathrm{D}$ values are larger than the $2 \mathrm{D}$ ones at the same locations, which is more pronounced for $\dot{\gamma}_{\mathrm{a}}=48 \mathrm{~s}^{-1}$.

Before the distance of $2 \mathrm{~mm}$, the deviations increase with the flow distance with larger discrepancies between the two models especially for low aspect ratios $(\leq 2)$. After $\sim 2 \mathrm{~mm}$ away from the die exit, interestingly, the swell ratio $B_{3}$ keeps almost constant for an aspect ratio of 1 (with the same final value as for $B_{1}, 1.3$; see Table III). For the other flow cells, it does not grow monotonously with the flow distance. Instead, an extrudate shrinkage behavior following the fast initial swelling is observed until the final equilibrium swelling positions in Figs. 5(b) and 5(d). For instance, within a short distance

TABLE III. Final (f) values for $B_{1}, B_{2}$, and $B_{3}$ at $\dot{\gamma}_{\mathrm{a}}=48 \mathrm{~s}^{-1}\left(200^{\circ} \mathrm{C}\right.$; PP extrudate; Table II). Also given are the differences; $\Delta B_{31}$ is essential in the secondary shrinkage explanation.

\begin{tabular}{llllcc}
\hline \hline $\begin{array}{l}\text { Aspect } \\
\text { ratio }\end{array}$ & $\begin{array}{c}\text { Final } \\
B_{1, f}\end{array}$ & $\begin{array}{c}\text { Final } \\
B_{2, f}\end{array}$ & $\begin{array}{c}\text { Final } \\
B_{3, f}\end{array}$ & $\begin{array}{c}\Delta B_{32, f}= \\
B_{3, f}-B_{2, f}\end{array}$ & $\begin{array}{c}\Delta B_{31, f}= \\
B_{3, f}-B_{1, f}\end{array}$ \\
\hline $1: 1$ & 1.3 & 1.145 & 1.3 & 0.155 & 0 \\
$1.5: 1$ & 1.284 & 1.06 & 1.309 & 0.249 & 0.025 \\
$2: 1$ & 1.26 & 0.99 & 1.31 & 0.32 & 0.05 \\
$5: 1$ & 1.22 & 0.86 & 1.31 & 0.45 & 0.09 \\
$10: 1$ & 1.19 & 0.81 & 1.294 & 0.484 & 0.104 \\
$20: 1$ & 1.15 & 0.78 & 1.288 & 0.508 & 0.138 \\
$2 \mathrm{D}$ & 0 & $1.314^{\mathrm{a}}$ & 1.314 & 0 & 1.314 \\
\hline
\end{tabular}

${ }^{\mathrm{a}}$ Not calculated but a consequence of the $2 \mathrm{D}$ assumption. after the exit, the swell behavior with an aspect ratio of 20 agrees well with that for the $2 \mathrm{D}$ flow. Then, with a further distance, a deviation is noticed because of the "secondary" shrinkage flow in the middle height direction (see also right extra subplots). More generally, the secondary shrinkage flows (symbols at higher positions in Fig. 5) in the middle height direction cannot be observed in the simplified steady $2 \mathrm{D}$ viscoelastic flow over the whole swell evolution. In addition, the differences of the swell ratio at various aspect ratios tend to become smaller with further flow distance. This can be attributed to the rather similar viscoelastic behavior of flows as the apparent shear rates are maintained the same for various aspect ratios. Such feature mainly determines the final equivalent swell ratio according to Eq. (11). Overall, the mismatches of the $2 \mathrm{D}$ modeling results highlight the effect of the side confining wall on the flow field (as confirmed in Secs. III B and III C) and thus the need of 3D simulations with both increases and decreases for swelling ratios along the distance.

This less detailed information with 2D models and the absence of shrinkage is in agreement with the general trends in most modeling studies. $26,56,57$ An exception is the modeling work of Comminal et al., ${ }^{53}$ which reported a small secondary shrinkage in the extrudate height direction after the initial swelling for the $2 \mathrm{D}$ planar viscoelastic flow. Relaxation of the negative normal stress component, $\tau_{y y}$, on the extrudate surface layer was put forward as the trigger. Recently, Toukhtarian et al. ${ }^{58}$ also revealed a shrinkage flow following the extrudate swell for 2D simplified transient planar Newtonian flow upon varying the die gap. It is attributed to the increasing volumetric flow rate because of the sudden decrease in the die gap. In the present work, it seems thus logical to assume that these triggers are absent (or counteracted) and an explanation needs to be sought in the $3 \mathrm{D}$ effect. In this respect, our previous $3 \mathrm{D}$ simulations for a die with fixed dimensions (aspect ratio of 10) pointed out that the swell ratio in the extrudate width direction increases in a much lower rate 
than the swell ratio in the height direction until an equilibrium is reached in both directions. ${ }^{44}$

To obey the volumetric conversation, a shrinkage flow is therefore expected in the extrudate height direction with several influencing factors. One factor is the difference of the final equilibrium width swell ratio $B_{1}$ and the final middle height swell ratio $B_{3}$ with a higher aspect ratio resulting in a larger difference, as shown in Table III. Another factor is the different growing rates of both swell ratios with the flow distance. For example, for an aspect ratio of 1 , no shrinkage flow is observed because the final $B_{1}$ is equal to the final $B_{3}$ (with a small drop for the final $B_{2}$ ). Increasing the aspect ratio results not only in a smaller final $B_{1}$ than $B_{3}$ but also in a smaller growing rate of $B_{1}$. Therefore, the synergistic effect of the aforementioned factors contributes to the secondary shrinkage flow in the height direction. In contrast, for the "ideal" $2 \mathrm{D}$ planar flow, the width of the flow channel is assumed to be infinite. This implies that the difference between $B_{1}$ and $B_{3}$ is too large (last column in Table III) to induce secondary shrinkage flow in the (middle) height direction, taking into account that contract flow at the edge cannot be accounted for in such modeling.

A global view of the final PP extrudate shapes (full black lines) from various dies (full red lines) is found in Fig. $6\left(\dot{\gamma}_{\mathrm{a}}=48 \mathrm{~s}^{-1}\right)$. In agreement with the analysis of the evolution profiles of the extrudate swell ratio (Figs. 4 and 5 and Table III) on an overall basis, higher aspect ratios result in a larger anisotropy degree of extrudate swell ratio upon considering jointly the height and width direction variations. Absolute variations up to $30 \%$ and relative variations of $10 \%$ can be identified, which explains the significant variation of the evolution of the area swell ratio $B_{4}$, shown in Fig. S3 in the supplementary material. For example, this ratio decreases from 1.55 to 1.417 by increasing the aspect ratio from 1 to 20 . Additionally, in any case, the $3 \mathrm{D}$ description is needed as illustrated by the deviation with the $2 \mathrm{D}$ simulation results (blue dotted-dashed lines) in Fig. 6.

\section{B. Relation aspect ratio and velocity fields}

\section{Axial velocity component $v_{x}$}

It is expected that the changes to the velocity field by varying the aspect ratios of the flow channels affect the extrudate swell behavior. Figure S4 in the supplementary material shows that as the aspect ratio increases, the velocity profile of the central flow along the width direction differs gradually from its analog along the height direction. For very high aspect ratios (e.g., 10 and 20), the velocity profile ( $y$ direction) overlaps the $2 \mathrm{D}$ flow profile. This means that in these die geometries, the $3 \mathrm{D}$ effect on the flow behavior in the $x-y$ plane $(z=0 \mathrm{~mm})$ in the die can be ignored.

However, as shown in Fig. 7, it is not safe to conclude that the $3 \mathrm{D}$ effect on the extrudate swell behavior out of the flow cell can be neglected as well. Figure 7 (a) compares the velocity profiles of the centerline flow along the flow direction with different aspect ratios at $\dot{\gamma}_{\mathrm{a}}=48 \mathrm{~s}^{-1}$. The axial velocity $v_{x}$ is scaled by the corresponding 2D average velocity value $v_{x a-2 D}$. A strong effect of the aspect ratio on the $v_{x}$ rearrangement behavior is observed. In agreement with the swelling data, increasing the aspect ratio causes a larger final equilibrium $v_{x}$ value. It is therefore not surprising that the simplified 2D flow has the largest equilibrium $v_{x}$ since there is no swell behavior in the width direction. For small aspect ratios $(\leq 2)$, the $v_{x}$ rearrangement behavior accomplishes within a short distance. From Fig. 7 (b), it follows that a higher aspect ratio postpones the position where the departure of the $v_{x}$ profile from that of $2 \mathrm{D}$ flow starts. For the aspect
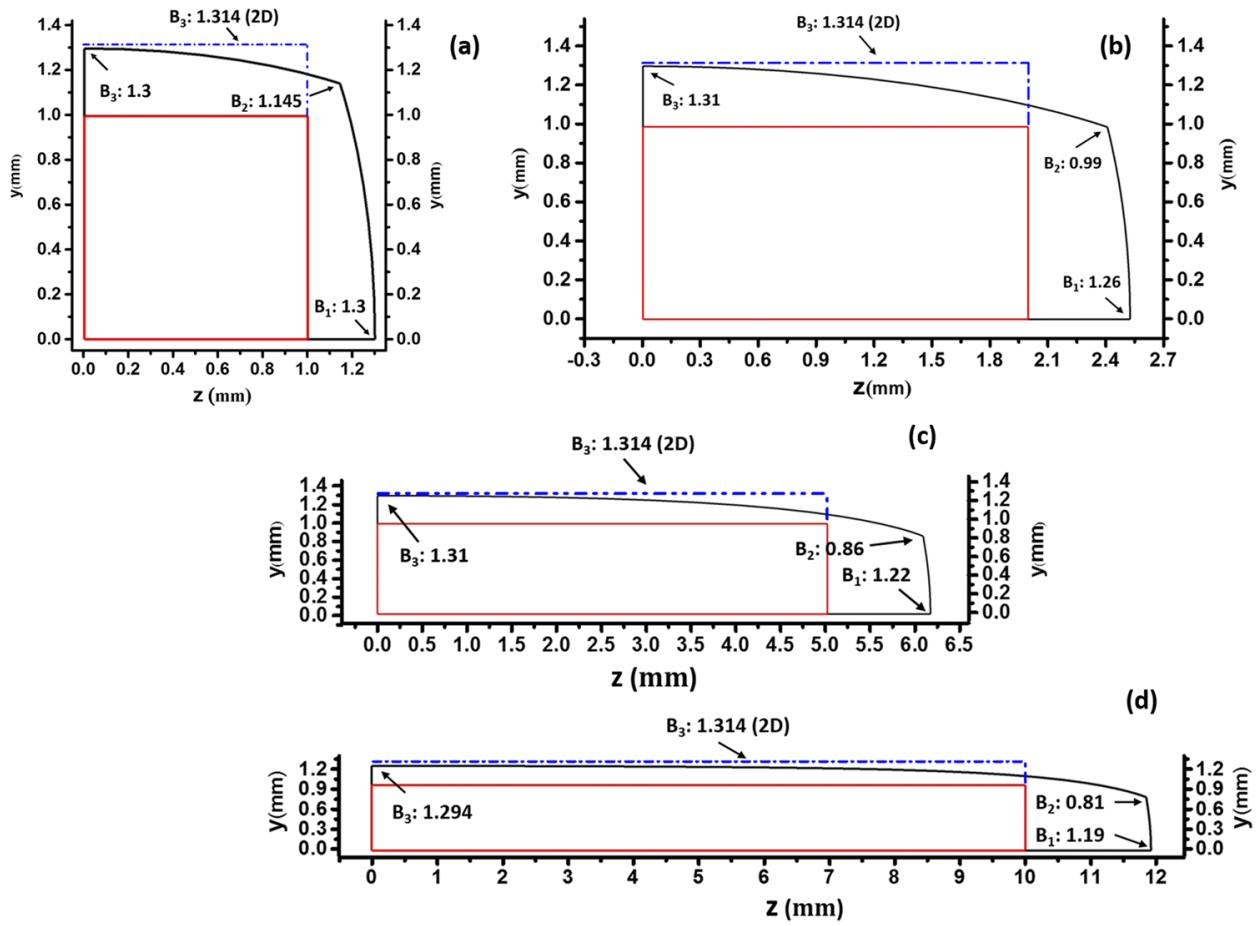

FIG. 6. Comparison of the final extrudate profiles (black solid lines) from slit dies (red solid lines) with different aspect ratios: (a): 1:1, (b): 2:1, (c): 5:1, and (d): 10:1 (Table II) at $\dot{\gamma}_{\mathrm{a}}=48 \mathrm{~s}^{-1}$. For comparison, also the simplified 2D results are included (blue dotted-dashed lines). Note that only one quarter is shown due to symmetry. Absolute changes up to $30 \%$ and relative changes in various directions of $10 \%$ can be observed. 

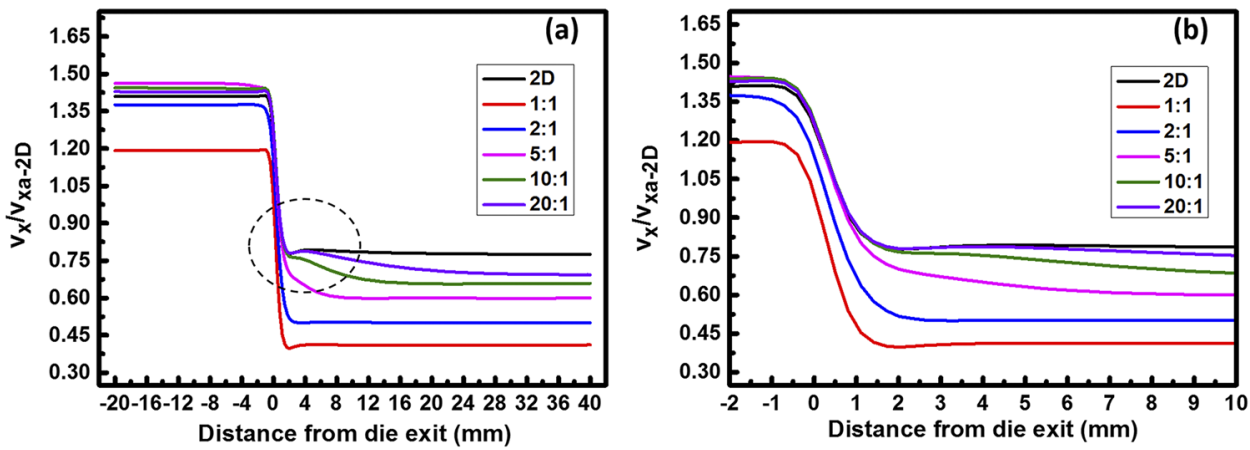

FIG. 7. Velocity profiles of centerline PP flow $(y=0$ and $z=0 \mathrm{~mm})$ at $\dot{\gamma}_{\mathrm{a}}=48 \mathrm{~s}^{-1}$ (Table II) through the dies with different aspect ratios along the flow direction $x$ Right: zoom in ratio of 20, the $v_{x}$ rearrangement behavior is almost the same as that of the 2D flow until a distance of around $6 \mathrm{~mm}$ after the exit. Then, $v_{x}$ continues to drop slowly in the former case.

\section{Velocity components $v_{y}$ and $v_{z}$}

Due to the flow reorganization, the velocity components $v_{y}$ and $v_{z}$ in the transversal $(y)$ and lateral $(z)$ directions separately increase sharply from a vanishing value just upon exiting the die, as shown in Fig. 8. Subplots (a) and (b) show the variations of $v_{y}$ along the extrudate free surface flow layer in the central plane $(\mathrm{z}=0 \mathrm{~mm})$ for $2 \mathrm{D}$ modeling and 3D flow with an aspect ratio of 1 and 20 at $\dot{\gamma}_{\mathrm{a}}=48 \mathrm{~s}^{-1}$. For an aspect ratio of $1, v_{y}$ falls fast to zero in comparison with the other two modeling cases. Seen in the inset in Fig. 8(a), the $v_{y}$ curves for 2D flow and 3D flow with an aspect ratio of 20 are almost the same, which correspond to the same swell behavior of the extrudate middle height for these two modeling cases in Fig. 5. For the former case, $v_{y}$ remains positive while it decreases to a negative value after a distance of $x=15 \mathrm{~mm}$ for the latter case. This accounts for the secondary shrinkage flow in the middle height direction for the aspect ratio of 20 in Fig. 5.

Figure 8 (c) shows the velocity $v_{y-e d g e}$ along the flow cross-line between the side and top flow planes of the extrudate free surface layers for modeling cases with an aspect ratio of 1 and 20. These velocity changes determine the swell behavior in the extrudate edge height direction. It is interesting to observe that the $v_{y \text {-edge }}$ values have different signs, namely, positive for an aspect ratio of 1 but negative for a value of 20 . Therefore, swell $\left(B_{2}>1\right)$ and shrinkage behavior $\left(B_{2}<1\right)$ can be observed for the two cases in Fig. 4(b), respectively. Figure 8 (d) presents the variations of the velocity component $v_{z}$ along the extrudate side free surface direction in the plane $y=0 \mathrm{~mm}$. It follows that the decrease in $v_{z}$ for an aspect ratio of 1 is significantly faster than that for an aspect ratio of 20 .

\section{Relation aspect ratio and stress field}

Generally, the normal stress relaxation development is also responsible for the evolution of the swell behavior of polymer
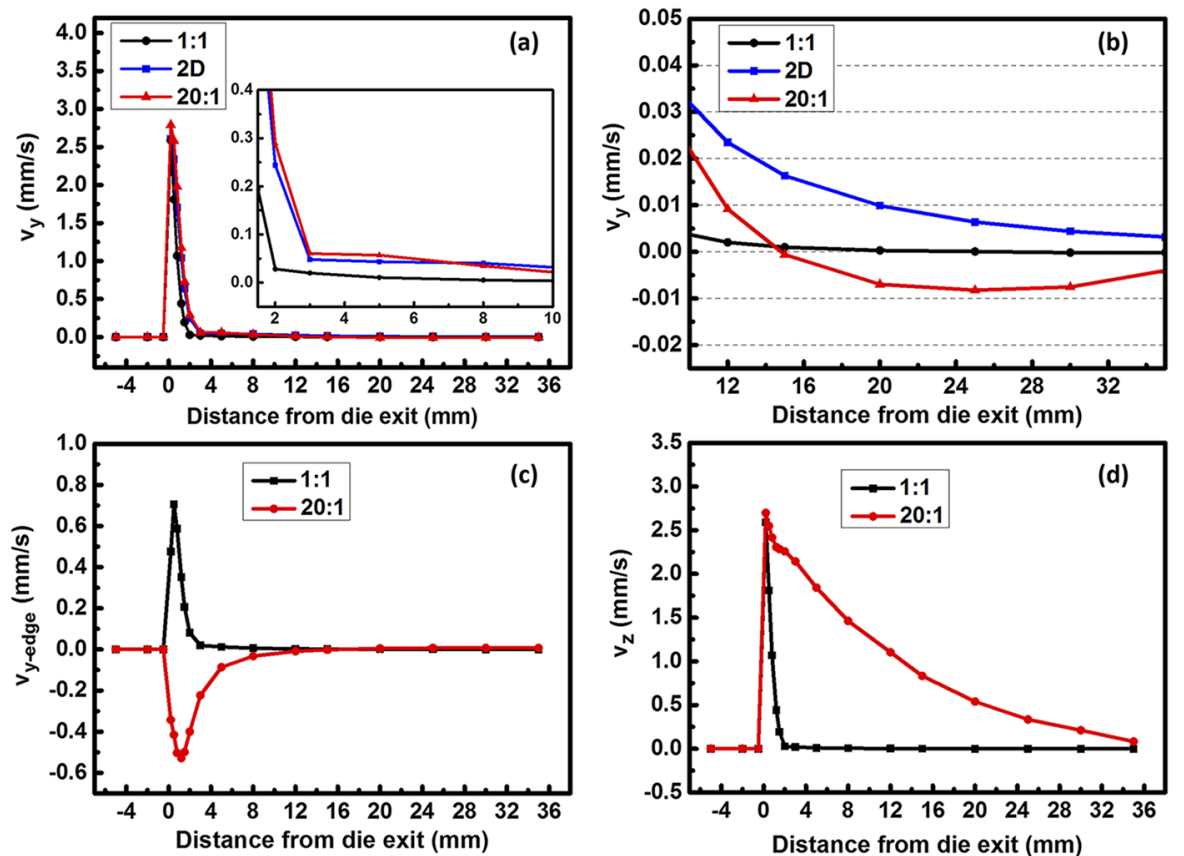

FIG. 8. (a) Velocity component $v_{y}$ along the die wall and free surface flow layer direction in the middle plane $z=0 \mathrm{~mm}$ for low and high aspect ratios with the 3D model, including a comparison with the ideal 2D flow; (b) is a close view of Fig. 8(a). (c) Velocity $v_{y \text {-edge }}$ along the flow cross-line between the side and top flow planes of extrudate free surface; (d) velocity component $v_{z}$ along the side die wall and free surface flow layer in the middle plane $y=0 \mathrm{~mm} . v_{y}, v_{y-e d g e}$, and $v_{z}$ codetermine the variations of extrudate middle height, edge height, and width, respectively, at $\dot{\gamma}_{\mathrm{a}}=48 \mathrm{~s}^{-1}$ 
fluids. ${ }^{44,53,59}$ Figure S5 in the supplementary material shows the calculated fully developed stress components at $x=-10 \mathrm{~mm}$ [shear stresses: $\tau_{y x}$ and $\tau_{z x}$; normal stress differences: $N_{x y}\left(\tau_{x x}-\tau_{y y}\right)$ and $\left.N_{x z}\left(\tau_{x x}-\tau_{z z}\right)\right]$ for all 3D and 2D flows at $\dot{\gamma}_{\mathrm{a}}=48 \mathrm{~s}^{-1}$. The related variations further confirm the $3 \mathrm{D}$ aspect due to the side confining wall. Figure 9 (a) compares $N_{x y}$ profiles for $3 \mathrm{D}$ flows with various aspect ratios and the corresponding $2 \mathrm{D}$ flow along the die wall and free surface direction in the symmetry planes. The calculated $N_{x y}$ profiles agree with the general trend in the literature ${ }^{18,26,60}$ with significantly different normal stress relaxation behavior due to the aspect ratio. Figures 9(b) and 9(c) give a close view of the stress relaxation away the die exit. For an aspect ratio of 1, a faster relaxation behavior is exhibited compared to that of the $2 \mathrm{D}$ counterpart, explaining the larger growing rate of the swell ratio $B_{3}$, and also the faster equilibrium swell positioning in Fig. 5. As the axial flow velocity with a low aspect ratio is smaller than that of the simplified 2D flow (Fig. 7), a longer time to reach the same distance, therefore leading to a larger influence of the normal stress relaxation. Hence, the middle height swell ratio $B_{3}$ increases much faster in comparison with the 2D counterpart in Fig. 5. In contrast, the situation is less dynamic for high aspect ratios (5 and 20). The stress relaxation of an aspect ratio of 20 is expected to agree better with that of the simplified $2 \mathrm{D}$ flow but still shows a bit faster relaxation.

Similarly, Fig. 9(d) compares the relaxation of $N_{x z}$ along the extrudate surface layer direction in the $x-z$ plane $(y=0 \mathrm{~mm})$, which is related to the swell behavior in the extrudate width direction. It is interesting to note that although the flow with an aspect ratio of 1 has a much larger $N_{x z}$ before die exit (see Fig. S5), it shows a faster normal stress relaxation. Besides the smaller axial velocity in Fig. 7, the relatively faster relaxation of the largely deformed polymer chains due to a larger deformation is involved as well. Increasing the aspect ratio slows down the $N_{x z}$ relaxation, thereby leading to a delayed influence on the swell behavior in the width direction. Hence, in an industrial context, the distance between the cooling system (e.g., water tank) and the die exit should be longer for a die with a large aspect ratio to minimize remaining internal stress, which can lead to a higher product quality.

\section{Parametric study regarding nonlinear PTT parameters}

The PTT parameters in Table I reflect the polymer melt rheological properties, which have an influence on the flow behavior in and out of the die. ${ }^{9}$ In essence, the parameters $\varepsilon_{i}$ and $\xi_{i}$ ( $i$ the mode) predominantly determine the extensional and shear behavior of polymer melts. The parameter $\xi_{i}$ is also related to the second normal stress difference $\left(N_{y z} ; \tau_{y y}-\tau_{z z}\right)$ that controls the polymer backbone slippage for the nonaffine motion of the surrounding continuum. ${ }^{9,20,37}$ Due to the difficulty in measuring $N_{y z}$ data, ${ }^{61}$ the effect of $N_{y z}$ on the extrudate behavior of the polymer melts is a point of attention. Konaganti et al. ${ }^{34}$ highlighted that the effect of $N_{y z}$ is very limited on the extrudate swell behavior, and therefore, they ignored this effect by considering zero $\xi_{i}$ values for the multimode PTT model to describe the rheological properties of the HDPE melt. On the other hand, Carrot et al..$^{14}$ reported that a real agreement of the extensional and shearing behavior of the polymer melts cannot be achieved by using a single $\varepsilon_{i}$ or $\xi_{i}$ parameter. Also our previous work on PP melts with a fixed aspect ratio indicates that both parameters are needed. Hence, it is worthwhile to further explore their relevance.
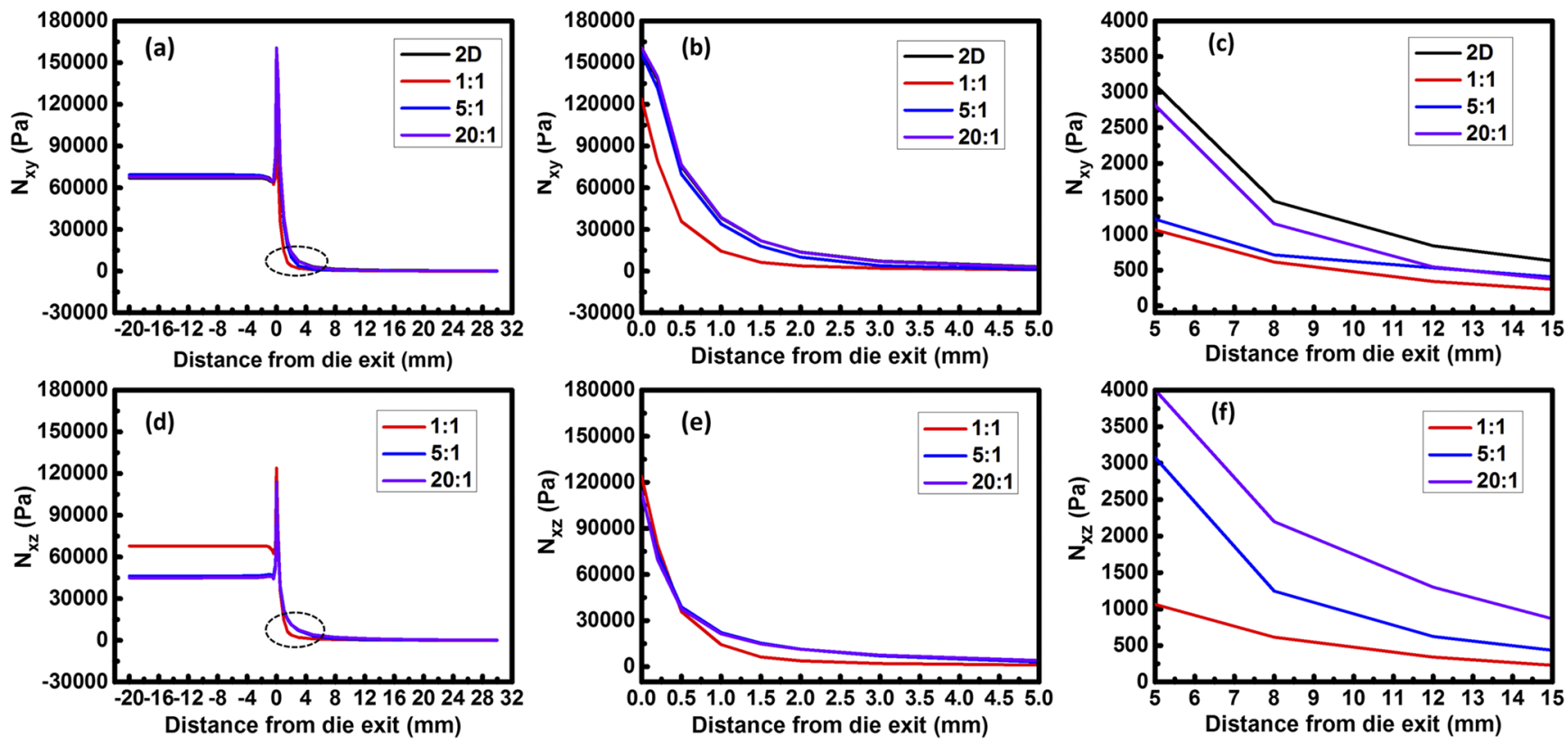

FIG. 9. Comparison of normal stress difference $N_{x y}$ [top row; (a)-(c); (a): full image; (b) and (c): close view] and $N_{x z}$ [bottom row; (d)-(f); (d): full image; (e) and (f): close view] along the wall and free surface layer at $\dot{\gamma}_{\mathrm{a}}=48 \mathrm{~s}^{-1}$. 
For simplicity, in this parametric study, the linear parameters $\left(\lambda_{i}\right.$ and $\left.\eta_{i}\right)$ are maintained constant in Table I. The aspect ratio of the flow channel is fixed at 10 and $\dot{\gamma}_{\mathrm{a}}=48 \mathrm{~s}^{-1}$. Preliminary screening showed that the parameters $\varepsilon$ and $\xi$ of mode 4 or mode 1 (the longest and shortest relaxation time) cannot have an obvious influence on the extrudate swell. For mode 4, the reason is likely that most elastic energy stored in the deformed polymer molecular chains has faded away before such long relaxation time, at least at the selected $\dot{\gamma}_{\mathrm{a}}$. For mode 1 , the selected deformation rate is simply not covered by mode 1 of the PTT model, which is mainly used to control the rheological properties of the melt at a rather high shear rate range. This is in agreement with literature data. ${ }^{28}$ Therefore, the impact of the PTT parameters of mode 3 and mode 2 on the extrudate swell behavior is only focused on.

Figure 10 depicts the simulated width and height swell ratio $B_{1}$ and $B_{3}$ with the $\xi$ range of $0.05-0.8$ in individual mode 3 [top row; (a)-(c)] and mode 2 [bottom row; (d)-(f)] for 3D and 2D configurations. Note that the values in Table I are inside this range and are denoted as reference values for simplicity. Figure 10(a) shows that the width swell ratio $B_{1}$ strongly depends on $\xi_{3}$, with a larger $\xi_{3}$ resulting in smaller $B_{1}$. For $B_{3}$ in Fig. 10(b), increasing $\xi_{3}$ from 0.2 to 0.8 decreases the swell ratio $B_{3}$ as well, consistent with the report of Goublomme et al. ${ }^{62}$ However, decreasing $\xi_{3}$ from 0.2 to 0.05 results in a smaller $B_{3}$ value, which is attributed to the strong "secondary" shrinkage flow in the latter case. Goublomme et al. ${ }^{62}$ implicated that $N_{y z}$ has a major influence on the swell ratio but also mentioned that a modest decrease in the uniaxial extensional viscosity caused by changing the material parameter should not be ignored. Consistent with this statement, Fig. 2 shows that a larger $\xi$ value results in a rather slight decrease in $N_{x y}$ and elongational viscosity values. In contrast, $\left|N_{y z}\right|$ increases significantly.

Additionally, as mentioned above, $B_{3}$ profiles for $\xi_{3}=0.05$ and 0.8 in Fig. 10(b) are rather different. The significant shrinkage flow follows the initially large swell behavior in the former case, which cannot be observed in the latter. This indicates that the $3 \mathrm{D}$ effect of the geometric channels on the extrudate height swell behavior depends on the material parameters of PTT model as well. It is important to realize that for $\xi_{3}=0.05$ and 0.8 , the difference between the final $B_{3}$ and $B_{1}$ values is 0.05 and 0.16 , respectively. This again confirms that the difference between the swell behavior in the (middle) extrudate height and width direction significantly contributes to the secondary shrinkage flow in the extrudate height direction (cf. Table III). For the simplified 2D flow in Fig. 10(c), the $B_{3}$ value increases monotonically with the flow distance without a shrinkage flow.

The material parameter $\varepsilon$ shows a large influence on the elongational viscosity during melt deformations shown in Fig. 2, and in case it starts to vanish, the elongational viscosity becomes unbounded. ${ }^{63}$ Figures 11(a), 11(b), 11(d), and 11(e) compare the calculated extrudate swell ratios with various $\varepsilon_{2}$ (bottom row) and $\varepsilon_{3}$ (top row) values from 0.05 to 0.65 . It is shown that both the width swell ratio $B_{1}$ and the height swell ratio $B_{3}$ decrease with increasing $\varepsilon$ value. To achieve a uniform velocity for each flow layer out of the die, the velocity difference between two flow layers leads to an extensional effect on the flow layers near the die exit, especially the flow layer close to the die wall. Formally increasing the $\varepsilon$ value decreases the elongational viscosity of the polymer flow, indicating the larger extensional effect applied on the polymer molecules
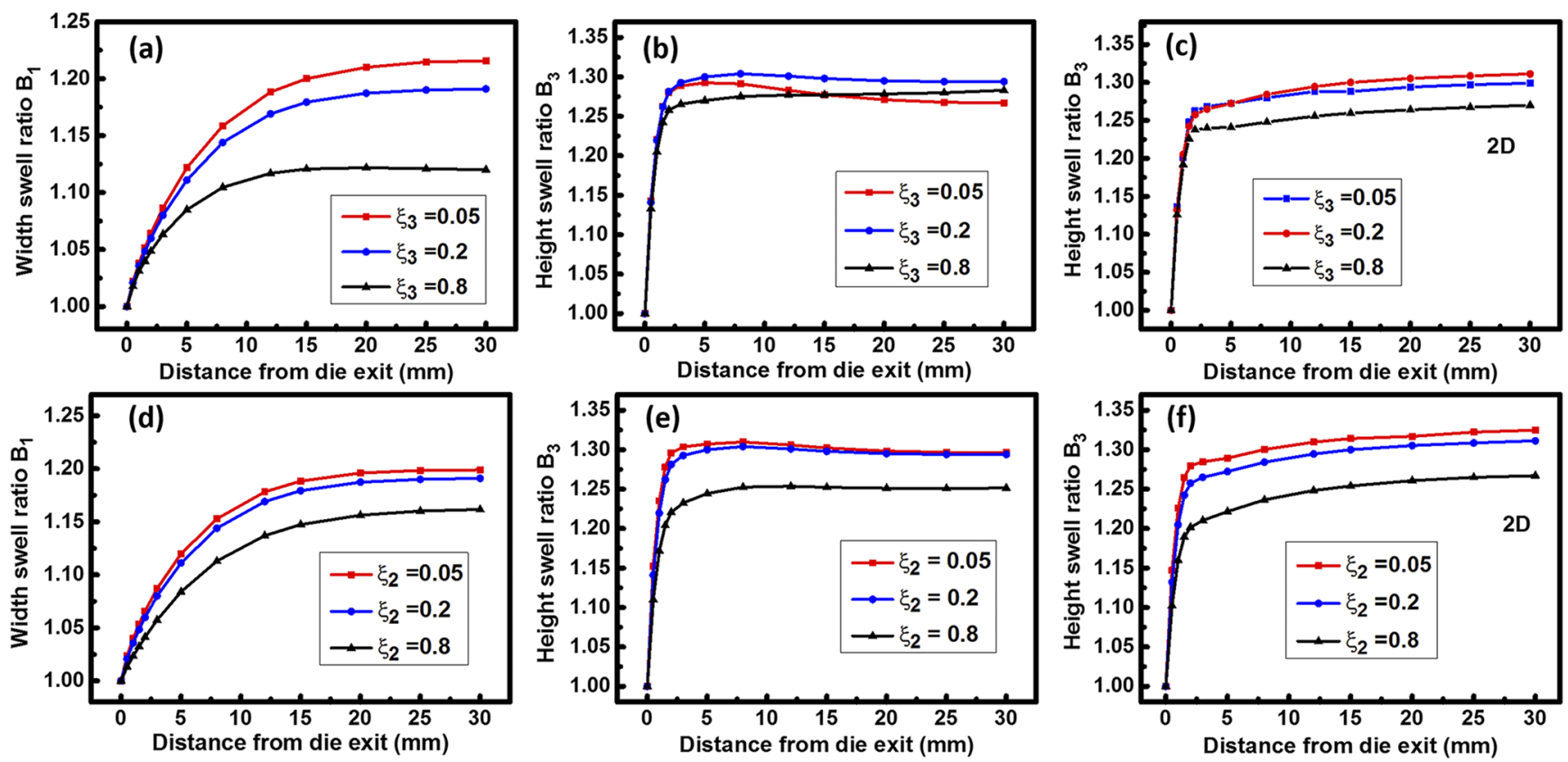

FIG. 10. Effect of the parameter $\xi$ in mode $3[(a)-(c)]$ and mode $2[(d)-(f)]$ of PTT model (other parameters as in Table I) on the extrudate swell ratio $B_{1}$ and $B_{3}$ with $3 D[(a)$ and $(\mathrm{b})$, and $(\mathrm{d})$ and $(\mathrm{e})]$ and $2 \mathrm{D}[(\mathrm{c})$ and $(\mathrm{f})]$ simulations (aspect ratio of 10$)$. 

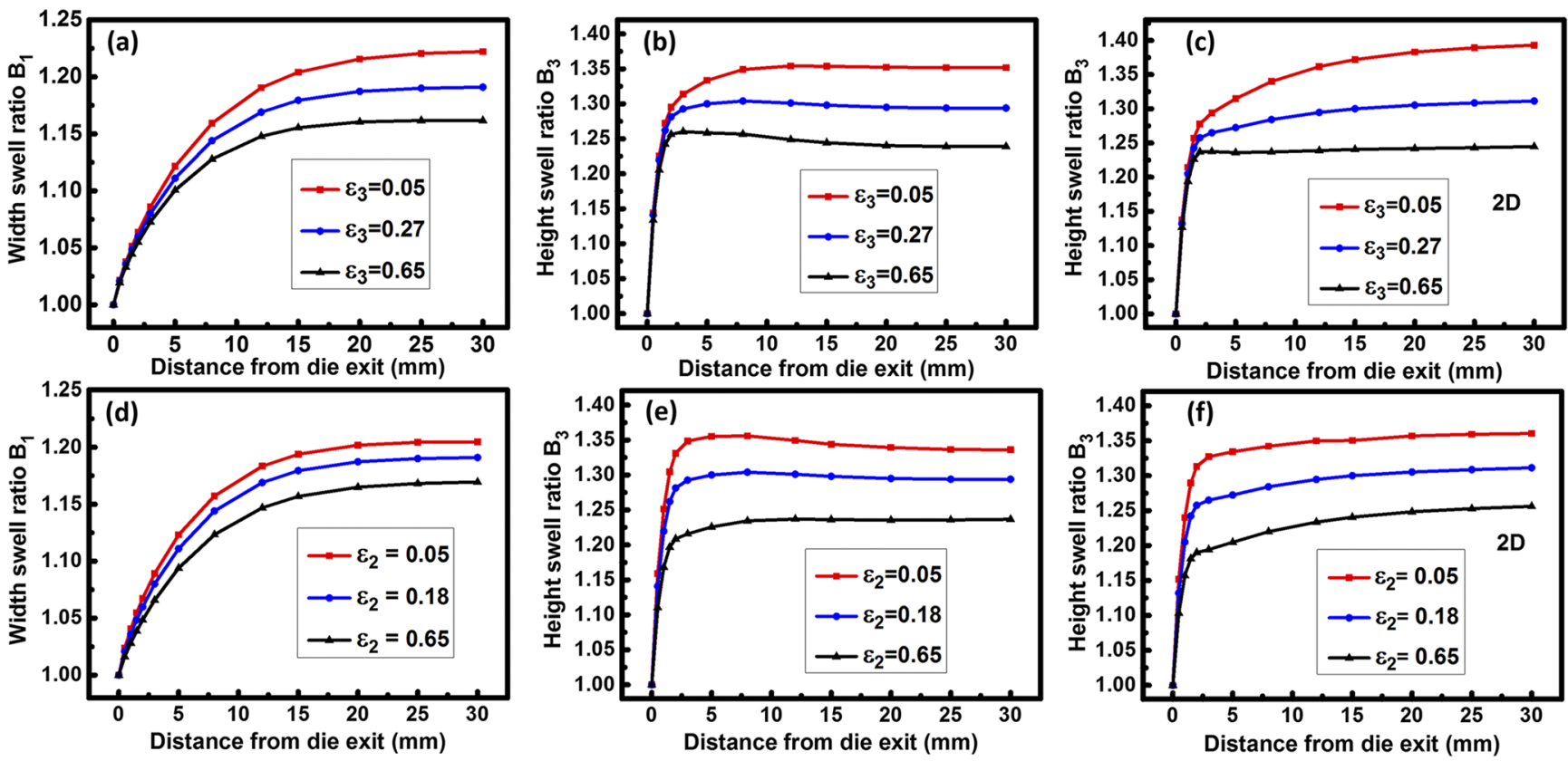

FIG. 11. Effect of the parameter $\varepsilon$ in mode $3[(a)-(c)]$ and mode $2[(d)-(f)]$ of the PTT model on the extrudate width swell ratio $B_{1}$ and height swell ratio $B_{3}$ with $3 D[(a)$, (b), (d), and $(\mathrm{e})]$ and $2 \mathrm{D}[(\mathrm{c})$ and $(\mathrm{f})]$ simulations. Other parameters are as in Table $\mathrm{I}$.

until the flow becomes stable. Therefore, the shrinkage of the flow streamline outside the die is expected, explaining the decrease in swell ratio $B_{1}$ and $B_{3}$. In addition, a comparison of the results in Figs. 11(b) and 11(e) shows that the secondary shrinkage flow at a larger distance in the extrudate height direction is related to the $\varepsilon$ values and cannot be observed in the $2 \mathrm{D}$ flow for which increases are always obtained. These effects are shown in Figs. 11(c) and 11(f), confirming the relevance of material parameters for detailed 3D simulations.

\section{CONCLUSIONS}

The influence of the slit die geometry on the extrudate flow behavior of neat PP melt with aspect ratios ranging from 1 to 20 is investigated via numerical simulations, considering the differential viscoelastic multimode PTT model with parameters taken from literature. To verify the impact of the die geometry, the $3 \mathrm{D}$ simulation results are compared to the simplified 2D case considering constant apparent shear rates. In any case, the $3 \mathrm{D}$ model is needed as die swell/shrinkage is multidimensional with absolute variations up to $30 \%$ and relative variations of $\sim 10 \%$. Hence, future process control and die design should be based on $3 \mathrm{D}$ interpretations.

Increasing the aspect ratio decreases the width swell ratio $B_{1}$ and the edge height swell ratio $B_{2}$ and leads to a shift in the onset of the axial equilibrium swelling position to a further distance. The edge height swell ratio $B_{2}$ can become even negative, leading to a contract flow. The middle height swell ratio $B_{3}$ profiles for the $3 \mathrm{D}$ flow significantly deviate from that of the $2 \mathrm{D}$ simulation. Just after the die exit, the $B_{3}$ values are larger than the $2 \mathrm{D}$ value and then a decrease in the $B_{3}$ values occurs, except for the aspect ratio of 1 , for which a $2 \mathrm{D}$ model is irrelevant. This phenomenon of a secondary shrinkage is not eliminated even if the aspect ratio is increased to the highest value of 20 .

The mechanism for the above phenomenon is illustrated by the flow velocity and stress fields. A significant deviation of the 3D configurations from the corresponding $2 \mathrm{D}$ one inside and outside the die is generally noted. Increasing the aspect ratio of the flow cells narrows the discrepancy but cannot vanish the 3D effect. For sure, the deviation out of the die still cannot be neglected, even if the flow behavior in the die ( $y$ direction) seems almost the same as that of the $2 \mathrm{D}$ flow with an aspect ratio higher than 2 . The larger axial velocity $v_{x}$ for a larger aspect ratio outside the die contributes to a slower normal stress relaxation, thereby a slower swell behavior delaying the swelling equilibrium position. This is also in agreement with the variations of the other velocity components $v_{y}$ and $v_{z}$.

A parametric study reveals that the width swell ratio $B_{1}$ largely decreases with increasing $\xi$ which is related to the second normal stress difference. An increasing $\varepsilon$ controlling the elongational viscosity decreases both the width well ratio $B_{1}$ and the height swell ratio $B_{3}$. In addition, the secondary shrinkage flow becomes large with decreasing $\xi$ but still cannot be observed in the corresponding 2D flow. This implies that the $3 \mathrm{D}$ effect on the extrudate swell behavior depends not only on the geometric characteristics of the flow channel but also on the melt material parameters. From this perspective, the impact of the 3D effect on the extrudate swell behavior is expected to vary if the polymer melts are modified or are combined with fillers. Such variations will be included in future work by the authors. 


\section{SUPPLEMENTARY MATERIAL}

See supplementary material for the complete mesh pattern and the check for the mesh-independence, the extrudate area swell data, and the flow behavior in the die (Figs. S1-S5).

\section{ACKNOWLEDGMENTS}

D.T. appreciates funding from the China Scholarship Council (Grant No. 201606240114) for the Ph.D. study at Ghent University.

\section{REFERENCES}

${ }^{1}$ T. Wieme, D. Tang, L. Delva, D. R. D'hooge, and L. Cardon, “The relevance of material and processing parameters on the thermal conductivity of thermoplastic composites," Polym. Eng. Sci. 58, 466 (2018).

${ }^{2}$ T. Wieme, L. Duan, N. Mys, L. Cardon, and D. D'hooge, "Effect of matrix and graphite filler on thermal conductivity of industrially feasible injection molded thermoplastic composites," Polymers 11, 87 (2019).

${ }^{3}$ S. Velayudhan, P. Ramesh, M. Sunny, and H. Varma, "Extrusion of hydroxyapatite to clinically significant shapes," Mater. Lett. 46, 142 (2000).

${ }^{4} \mathrm{E}$. Miller and J. P. Rothstein, "Control of the sharkskin instability in the extrusion of polymer melts using induced temperature gradients," Rheol. Acta 44, 160 (2004).

${ }^{5}$ M. Ebrahimi, T. Tomkovic, G. Liu, A. A. Doufas, and S. G. Hatzikiriakos, "Melt fracture of linear low-density polyethylenes: Die geometry and molecular weight characteristics," Phys. Fluids 30, 053103 (2018).

${ }^{6} \mathrm{C}$. Beverly and R. Tanner, "Numerical analysis of extrudate swell in viscoelastic materials with yield stress," J. Rheol. 33, 989 (1989).

${ }^{7}$ C. Sirisinha, "A review of extrudate swell in polymers," ScienceAsia 23, 259 (1997).

${ }^{8}$ J. Liang, C. Chen, T. Zhou, S. Zou, W. Huang, C. Tsui, C. Tang, and V. MiškovićStanković, "Melt extrudate swell behavior of multi-walled carbon nanotubes filledpolypropylene composites," Polym. Compos. 38, 2433 (2017).

${ }^{9}$ H. Tian, D. Zhao, M. Wang, G. Jin, and Y. Jin, "Study on extrudate swell of polypropylene in double-lumen micro profile extrusion," J. Mater. Process. Technol. 225, 357 (2015).

${ }^{10} \mathrm{~N}$. Neves, A. Pontes, and A. Pouzada, "Experimental validation of morphology simulation in glass fibre reinforced polycarbonate discs," J. Reinf. Plast. Compos. 20, 452 (2001)

${ }^{11}$ L. Ferrás, A. Afonso, J. Nóbrega, and F. Pinho, "A numerical and theoretical study on viscoelastic fluid slip flows," Phys. Fluids 29, 053102 (2017).

${ }^{12} \mathrm{~T}$. Barborik and M. Zatloukal, "Effect of heat transfer coefficient, draw ratio, and die exit temperature on the production of flat polypropylene membranes," Phys. Fluids 31, 053101 (2019).

${ }^{13}$ B. Caswell and M. Viriyayuthakorn, "Finite element simulation of die swell for a Maxwell fluid," J. Non-Newtonian Fluid Mech. 12, 13 (1983).

${ }^{14}$ C. Carrot, J. Guillet, P. Revenu, and A. Arsac, Experimental Validation of Non Linear Network Models (Elsevier, 1996).

${ }^{15} \mathrm{~S}$. X. Huang and C. J. Lu, "Stress relaxation characteristics and extrudate swell of the IUPAC-LDPE melt," J. Non-Newtonian Fluid Mech. 136, 147 (2006).

${ }^{16}$ A. Goublomme, B. Draily, and M. Crochet, "Numerical prediction of extrudate swell of a high-density polyethylene," J. Non-Newtonian Fluid Mech. 44, 171 (1992).

${ }^{17}$ S. X. Huang, X. Chen, C. J. Lu, Z. L. Peng, C. R. Wan, X. He, and M. X. Sun, "Rheological characterizations and extrudate swell predictions of an LDPE melt by two KBKZ-type constitutive equations," J. Hydrodyn. 22, 872 (2010).

${ }^{18}$ V. K. Konaganti, M. Ansari, E. Mitsoulis, and S. G. Hatzikiriakos, "The effect of damping function on extrudate swell," J. Non-Newtonian Fluid Mech. 236, 73 (2016).

${ }^{19}$ V. K. Konaganti, M. Derakhshandeh, M. Ebrahimi, E. Mitsoulis, and S. G. Hatzikiriakos, "Non-isothermal extrudate swell," Phys. Fluids 28, 123101 (2016).

${ }^{20}$ N. P. Thien and R. I. Tanner, "A new constitutive equation derived from network theory," J. Non-Newtonian Fluid Mech. 2, 353 (1977).
${ }^{21}$ F. Sugeng, N. Phan-Thien, and R. Tanner, "A study of non-isothermal nonNewtonian extrudate swell by a mixed boundary element and finite element method," J. Rheol. 31, 37 (1987).

${ }^{22}$ Y. Mu, G. Zhao, H. Li, J. Liu, X. Xu, W. Mu, and A. Chen, "Measurement and simulation of low-density polyethylene extrudate swell through a circular die," Polym. Int. 58, 475 (2009).

${ }^{23}$ Y. Mu, G. Zhao, A. Chen, and X. Wu, "Modeling and simulation of threedimensional extrusion swelling of viscoelastic fluids with PTT, Giesekus and FENE-P constitutive models," Int. J. Numer. Methods Fluids 72, 846 (2013).

${ }^{24}$ Y. Mu, G. Zhao, and C. Zhang, "Numerical investigation of die geometry effect on LDPE annular extrudate swell," J. Appl. Polym. Sci. 117, 91 (2010).

${ }^{25} \mathrm{~J}$. Azaiez, R. Guénette, and A. Ait-Kadi, "Numerical simulation of viscoelastic flows through a planar contraction," J. Non-Newtonian Fluid Mech. 62, 253 (1996).

${ }^{26}$ C. Béraudo, A. Fortin, T. Coupez, Y. Demay, B. Vergnes, and J. Agassant, "A finite element method for computing the flow of multi-mode viscoelastic fluids: Comparison with experiments," J. Non-Newtonian Fluid Mech. 75, 1 (1998).

${ }^{27} \mathrm{~S}$. Tanoue and Y. Iemoto, "Effect of die gap width on annular extrudates by the annular extrudate swell simulation in steady-states," Polym. Eng. Sci. 39, 2172 (1999).

${ }^{28} \mathrm{~J}$. Schoonen, Determination of Rheological Constitutive Equations Using Complex Flows (Technische Universiteit Eindhoven Eindhoven, 1998).

${ }^{29}$ J. Guillet, C. Carrot, B. Kim, J. Agassant, B. Vergnes, C. Beraudo, J. Clermont, M. Normandin, and Y. Bereaux, Comparison between Experimental Data and Numerical Models (Elsevier, 1996).

${ }^{30}$ T. Tomkovic, E. Mitsoulis, and S. G. Hatzikiriakos, "Contraction flow of ionomers and their corresponding copolymers: Ionic and hydrogen bonding effects," Phys. Fluids 31, 033102 (2019).

${ }^{31}$ E. Behzadfar, M. Ansari, V. K. Konaganti, and S. G. Hatzikiriakos, "Extrudate swell of HDPE melts: I. Experimental," J. Non-Newtonian Fluid Mech. 225, 86 (2015).

${ }^{32}$ A. García-Rejón, R. DiRaddo, and M. Ryan, "Effect of die geometry and flow characteristics on viscoelastic annular swell," J. Non-Newtonian Fluid Mech. 60, 107 (1995).

${ }^{33}$ V. Konaganti, E. Behzadfar, M. Ansari, E. Mitsoulis, and S. Hatzikiriakos, "Extrudate swell of high density polyethylenes in slit (flat) dies," Int. Polym. Process. 31, 262 (2016).

${ }^{34}$ V. Konaganti, M. Ansari, E. Mitsoulis, and S. G. Hatzikiriakos, "Extrudate swell of a high-density polyethylene melt: II. Modeling using integral and differential constitutive equations," J. Non-Newtonian Fluid Mech. 225, 94 (2015).

${ }^{35}$ W. M. Verbeeten, G. W. Peters, and F. P. Baaijens, "Numerical simulations of the planar contraction flow for a polyethylene melt using the XPP model," J. NonNewtonian Fluid Mech. 117, 73 (2004).

${ }^{36}$ E. Mitsoulis and I. Argyropaidas, "Numerical simulation of contraction and expansion flows of Langmuir monolayers," J. Non-Newtonian Fluid Mech. 157, 163 (2009).

${ }^{37}$ N. Clemeur, R. Rutgers, and B. Debbaut, "Numerical simulation of abrupt contraction flows using the double convected Pom-Pom model," J. Non-Newtonian Fluid Mech. 117, 193 (2004).

${ }^{38} \mathrm{R}$. Ahmed, R. Liang, and M. Mackley, "The experimental observation and numerical prediction of planar entry flow and die swell for molten polyethylenes," J. Non-Newtonian Fluid Mech. 59, 129 (1995).

${ }^{39}$ A. Bogaerds, W. Verbeeten, G. Peters, and F. Baaijens, "3D viscoelastic analysis of a polymer solution in a complex flow," Comput. Methods Appl. Mech. Eng. 180, 413 (1999).

${ }^{40} \mathrm{~N}$. Clemeur, R. Rutgers, and B. Debbaut, "Numerical evaluation of three dimensional effects in planar flow birefringence," J. Non-Newtonian Fluid Mech. 123, 105 (2004).

${ }^{41}$ S. C. Xue, N. Phan-Thien, and R. Tanner, "Three dimensional numerical simulations of viscoelastic flows through planar contractions," J. Non-Newtonian Fluid Mech. 74, 195 (1998).

${ }^{42}$ S. Omowunmi and X. F. Yuan, "Modelling the three-dimensional flow of a semidilute polymer solution in microfluidics-on the effect of aspect ratio," Rheol. Acta 49, 585 (2010). 
${ }^{43}$ J. F. Schoonen, F. H. Swartjes, G. W. Peters, F. Baaijens, and H. E. Meijer, "A 3D numerical/experimental study on a stagnation flow of a polyisobutylene solution," J. Non-Newtonian Fluid Mech. 79, 529 (1998).

${ }^{44}$ D. Tang, F. H. Marchesini, D. R. D’hooge, and L. Cardon, "Isothermal flow of neat polypropylene through a slit die and its die swell: Bridging experiments and 3D numerical simulations," J. Non-Newtonian Fluid Mech. 266, 33 (2019).

${ }^{45}$ G. O. Aloku and X. F. Yuan, "Numerical simulation of polymer foaming process in extrusion flow," Chem. Eng. Sci. 65, 3749 (2010).

${ }^{46}$ L. F. C. Rodríguez, Modification of the PTT model and its application for the predictions of elongational viscosity of polypropylenes-Edición Unnica.

${ }^{47} \mathrm{P}$. Saramito and J. Piau, "Flow characteristics of viscoelastic fluids in an abrupt contraction by using numerical modeling," J. Non-Newtonian Fluid Mech. 52, 263 (1994).

${ }^{48} \mathrm{R}$. Guénette and M. Fortin, "A new mixed finite element method for computing viscoelastic flows," J. Non-Newtonian Fluid Mech. 60, 27 (1995).

${ }^{49}$ S. C. Xue, N. Phan-Thien, and R. Tanner, "Numerical study of secondary flows of viscoelastic fluid in straight pipes by an implicit finite volume method," J. NonNewtonian Fluid Mech. 59, 191 (1995).

${ }^{50}$ M. Ansari, S. G. Hatzikiriakos, and E. Mitsoulis, "Slip effects in HDPE flows," J. Non-Newtonian Fluid Mech. 167, 18 (2012).

${ }^{51}$ D. G. Baird, "First normal stress difference measurements for polymer melts at high shear rates in a slit-die using hole and exit pressure data," J. Non-Newtonian Fluid Mech. 148, 13 (2008).

${ }^{52}$ C. W. Macosko and R. G. Larson, Rheology: Principles, Measurements, and Applications (Wiley-VCH, 1994).

${ }^{53}$ R. Comminal, F. Pimenta, J. H. Hattel, M. A. Alves, and J. Spangenberg, "Numerical simulation of the planar extrudate swell of pseudoplastic and viscoelastic fluids with the streamfunction and the VOF methods," J. NonNewtonian Fluid Mech. 252, 1 (2018).
${ }^{54}$ R. I. Tanner, "A theory of die-swell revisited," J. Non-Newtonian Fluid Mech. 129, 85 (2005).

${ }^{55} \mathrm{M}$. Normandin, J. R. Clermont, J. Guillet, and C. Raveyre, “Three-dimensional extrudate swell experimental and numerical study of a polyethylene melt obeying a memory-integral equation," J. Non-Newtonian Fluid Mech. 87, 1 (1999).

${ }^{56} \mathrm{~V}$. Ganvir, A. Lele, R. Thaokar, and B. Gautham, "Prediction of extrudate swell in polymer melt extrusion using an Arbitrary Lagrangian Eulerian (ALE) based finite element method," J. Non-Newtonian Fluid Mech. 156, 21 (2009).

${ }^{57}$ V. Ganvir, B. Gautham, H. Pol, M. S. Bhamla, L. Sclesi, R. Thaokar, A. Lele, and M. Mackley, "Extrudate swell of linear and branched polyethylenes: ALE simulations and comparison with experiments," J. Non-Newtonian Fluid Mech. 166, 12 (2011).

${ }^{58}$ R. Toukhtarian, S. G. Hatzikiriakos, H. Atsbha, and B. Boulet, "Modeling polymer extrusion with varying die gap using Arbitrary Lagrangian Eulerian (ALE) method," Phys. Fluids 30, 093103 (2018).

${ }^{59}$ R. Tanner, “A theory of die-swell,” J. Polym. Sci., Part B: Polym. Phys. 8, 2067 (1970).

${ }^{60} \mathrm{~A}$. Al-Muslimawi, H. Tamaddon-Jahromi, and M. Webster, "Simulation of viscoelastic and viscoelastoplastic die-swell flows," J. Non-Newtonian Fluid Mech. 191, 45 (2013).

${ }^{61}$ J. M. Dealy and R. G. Larson, Nonlinear Viscoelasticity (Carl Hanser Verlag $\mathrm{GmbH} \& \mathrm{Co} . \mathrm{KG}, 2006$ ).

${ }^{62} \mathrm{~A}$. Goublomme and M. Crochet, "Numerical prediction of extrudate swell of a high-density polyethylene: Further results," J. Non-Newtonian Fluid Mech. 47, 281 (1993).

${ }^{63} \mathrm{G}$. Karapetsas and J. Tsamopoulos, "Steady extrusion of viscoelastic materials from an annular die," J. Non-Newtonian Fluid Mech. 154, 136 (2008). 